\title{
Gallbladder Neuroendocrine Carcinoma: A Case Report with Radiological-Pathological Correlation
}

\author{
Karamvir Chandel ${ }^{1} \quad$ Anupam Lal ${ }^{1} \quad$ Rohit K. Srinivas $^{1}$ \\ ${ }^{1}$ Department of Radiodiagnosis and Imaging, Postgraduate Institute \\ of Medical Education and Research, Chandigarh, India \\ 2 Department of Pathology, Postgraduate Institute of Medical \\ Education and Research, Chandigarh, India \\ 3 Department of Surgery, Postgraduate Institute of Medical Education \\ and Research, Chandigarh, India
}

J Gastrointestinal Abdominal Radiol ISGAR 2022;5:191-195.

\author{
Debajyoti Chatterjee $^{2}$ Vikas Gupta ${ }^{3}$
}

\begin{abstract}
Keywords

- gallbladder

- neuroendocrine tumor

- radiology
\end{abstract}

\author{
Address for correspondence Anupam Lal, MD, Department of \\ Radiodiagnosis and Imaging, Postgraduate Institute of Medical \\ Education and Research, Sector-12, Chandigarh 160012, India \\ (e-mail: lalpgimer@gmail.com).
}

\section{Introduction}

Adenocarcinomas are the most common malignant gallbladder (GB) neoplasms, accounting for $90 \%$ of all GB carcinomas. Squamous, small cell, adenosquamous, and undifferentiated epithelial cells are among the other epithelial cell types. Neuroendocrine tumors (NET) of the biliary system, specifically GB, are extremely rare, accounting for only $0.2 \%$ of all NETs. ${ }^{1}$ Maximum GBNETs are typically identified in pathological specimens of GB samples obtained at autopsy, following cholecystectomy, or following surgery for other suspected biliary pathology. ${ }^{2}$ It is thought that GBNETs develop from preexisting endocrine cells in the GB neck region or as a result of intestinal metaplasia of endocrine cells in the body and fundus. ${ }^{3,4}$ Because of its rarity, we present a case of squamous cell carcinoma of GB with neuroendocrine differentiation with some atypical imaging findings and its radio-pathological discussion.

\section{Case Report}

A 46-year-old normotensive, nondiabetic female patient presented with newly onset dull aching in the epigastrium and right hypochondrium for 1 month. There was no history of fever or jaundice. The abdomen was soft, non-tender, and non-distended upon examination. Ultrasound examination demonstrated distended GB, with the heterogeneous exoendophytic lesion of mixed echogenicity in the GB with associated asymmetric GB wall thickening (-Fig. 1A). A well-defined hyperechoic lesion was noted in the liver, adjacent to the GB fossa (-Fig. 1B). No intrahepatic biliary tree dilatation was noted. Liver size, echotexture, and outline published online February 24, 2022
DOI https://doi.org/ 10.1055/s-0042-1742676. ISSN 2581-9933.

\footnotetext{
(C) 2022. Indian Society of Gastrointestinal and Abdominal Radiology. All rights reserved.

This is an open access article published by Thieme under the terms of the Creative Commons Attribution-NonDerivative-NonCommercial-License, permitting copying and reproduction so long as the original work is given appropriate credit. Contents may not be used for commercial purposes, or adapted, remixed, transformed or built upon. (https://creativecommons.org/ licenses/by-nc-nd/4.0/)

Thieme Medical and Scientific Publishers Pvt. Ltd., A-12, 2nd Floor, Sector 2, Noida-201301 UP, India
} 

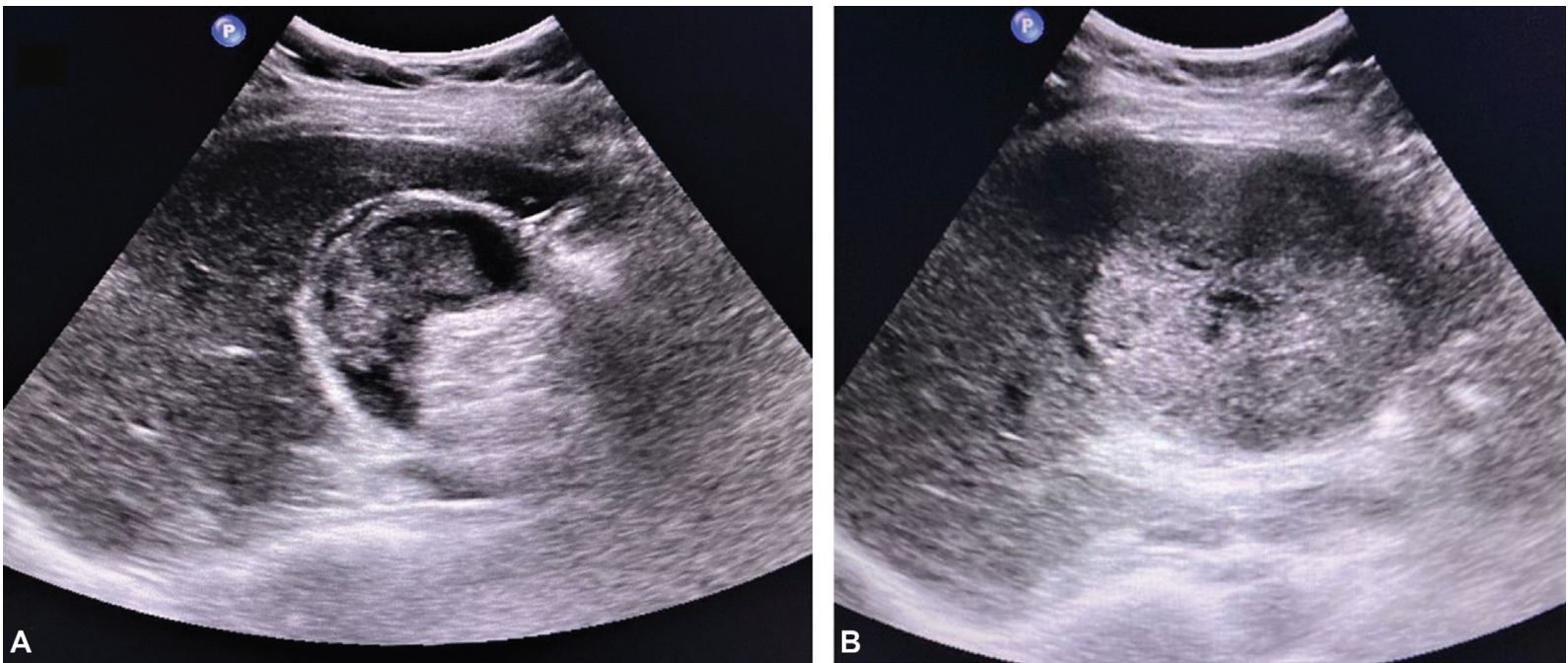

Fig. 1 Gray-scale ultrasonography demonstrates (A) polypoidal heteroechoic endophytic mass lesion in gallbladder (GB) with asymmetric GB wall thickening, and (B) well-defined heterogenous hyperechoic lesion in the adjacent liver contiguous with the GB mass.

were normal. The most likely diagnosis was GB carcinoma with hepatic extension. Her routine laboratory tests came back within normal limits.

Biphasic (arterial phase followed by venous phase) contrast-enhanced computed tomography (CECT) was done. In the arterial phase, a distended GB with an arterial hyperenhancing polypoidal mass lesion (-Fig. 2A), along with contiguous infiltration into adjacent left lobe segments IV A and IV B, and the presence of a relatively well-defined, arterial hyper-enhancing lesion, measuring approximately $7 \times 7.3 \times 6 \mathrm{~cm}$ ( - Fig. 2B), were observed. In the body region, there was associated asymmetric GB mural thickening. The hepatic component showed homogenous enhancement with washout in the portal venous phase compared with the rest of the liver ( - Fig. 2E and F). Few hypodense areas were noted within, which may be suggestive of necrosis. The hepatic lesion was inextricably linked to the GB lesion. The portal and hepatic veins were normal. Portocaval lymph nodal mass with a few other periportal enlarged lymph nodes was noted. The lymph nodes showed arterial enhancement in the arterial phase and homogenous enhancement in the venous phase, similar to the primary lesion ( $\mathbf{- F i g}$. $\mathbf{2 A}$ and $\mathbf{C}$ ). The radiological diagnosis was GB carcinoma with direct liver invasion and metastatic portocaval lymphadenopathy, with a
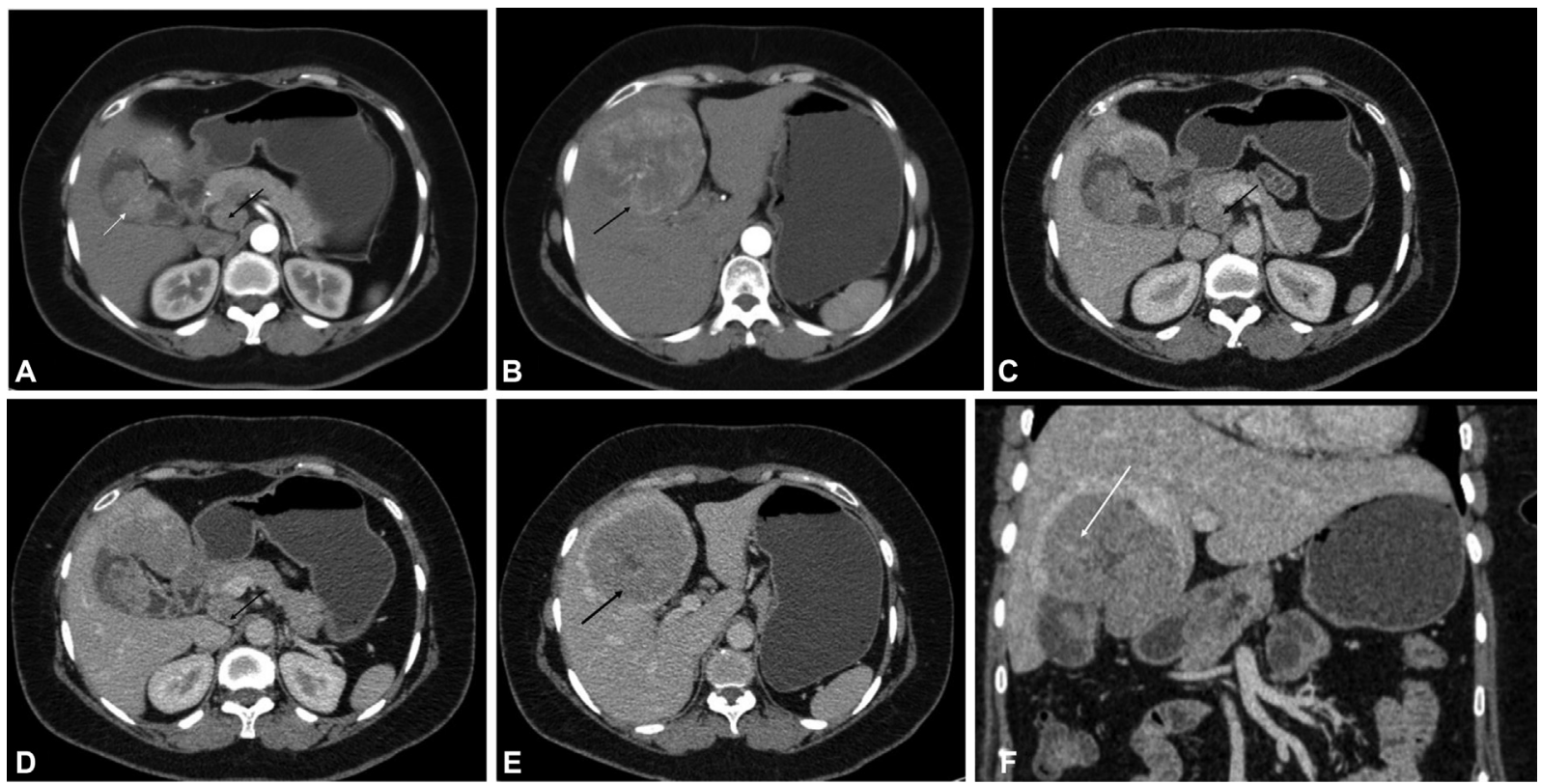

Fig. 2 Triple-phase contrast-enhanced computed tomography scan demonstrates: axial arterial phase (A) polypoidal heterogeneously enhancing endophytic mass lesion in gallbladder (GB) (white arrow) with asymmetric GB wall thickening. Enlarged lymph nodal mass in precaval location (black arrow), (B) well-defined heterogenous arterial hyper-vascular lesion in the adjacent liver contiguous with the GB mass (dashed black arrow). Venous phase (C) and (D) shows GB mass and lymph nodal mass (arrow), (E) hepatic lesion shows washout with enhancing peripheral rim (dashed black arrow), and coronal (F) venous phase image shows central hypodensity (dashed white arrow). 


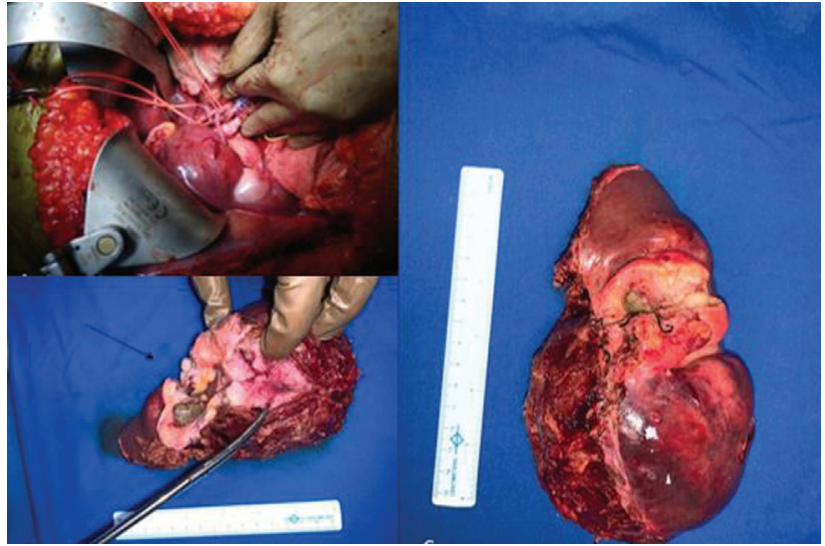

Fig. 3 Intraoperative images showing gallbladder (GB) mass and resected liver with $G B$ mass.

high possibility of neuroendocrine carcinoma due to arterial hypervascularity and washout. Differential diagnosis of arterial hyper-vascular hepatic malignancy (Hepatocellular carcinoma, fibrolamellar variant) with GB infiltration and lymph node metastasis was also given because of washout and capsular enhancement and a focal central hypodense region (-Fig. 2E and F) which might represent scar. Because the GB mass was polypoidal, it was also more suggestive of primary GB malignancy. Alpha-fetoprotein levels were normal, but chromogranin A levels were elevated $(128 \mathrm{~g} / \mathrm{mL}$ vs. normal $36.4 \mathrm{~g} / \mathrm{mL}$ ).

According to American Joint Committee on Cancer, the TNM classification of GB cancer staging in our case was T3N1M0. ${ }^{4}$ The patient underwent open extended cholecystectomy with major hepatectomy (segment IV B, V, and VI) (-Fig. 3). Gross

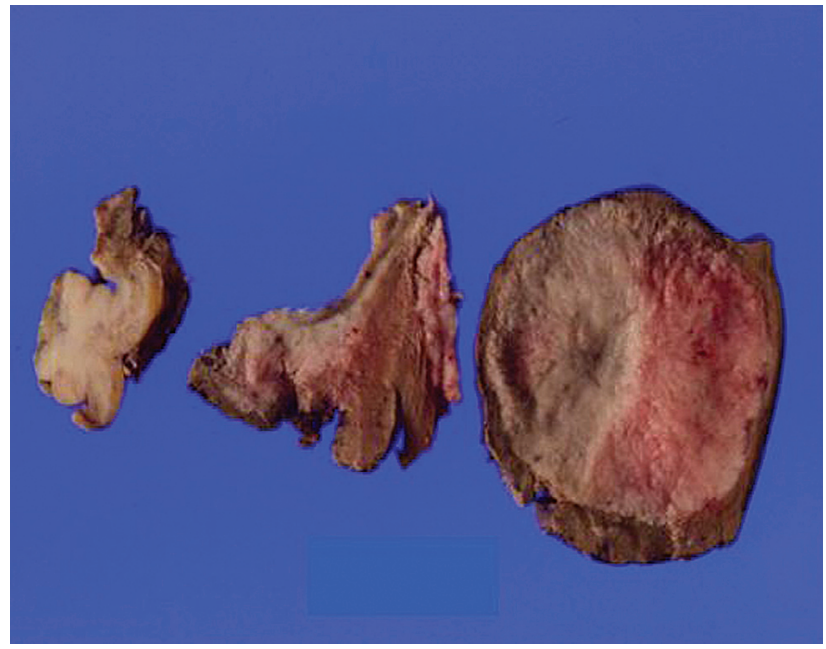

Fig. 4 Serially sliced gross specimen showing a tumor arising from the gall bladder and infiltrating the liver with a pushing margin.

specimen examination (- Fig. 4) showed firm exo-endophytic GB tumor with infiltrative margins and contiguous infiltration into the liver. Areas of necrosis and hemorrhages were seen within. The cystic duct was free of tumors. A conglomerated lymph nodal mass was seen along with other enlarged lymph nodes that were positive for tumor cells. On microscopic examination (-Fig. $\mathbf{5 A - C}$ ), a tumor arising from ulcerated GB mucosa was observed with tumor cells arranged in islands in the desmoplastic stroma. The tumor cells showed squamous differentiation with large polygonal cells, with the distinct cell membrane, central pleomorphic nuclei, coarse chromatin, and dense eosinophilic cytoplasm. A large area of the tumor (60\%)
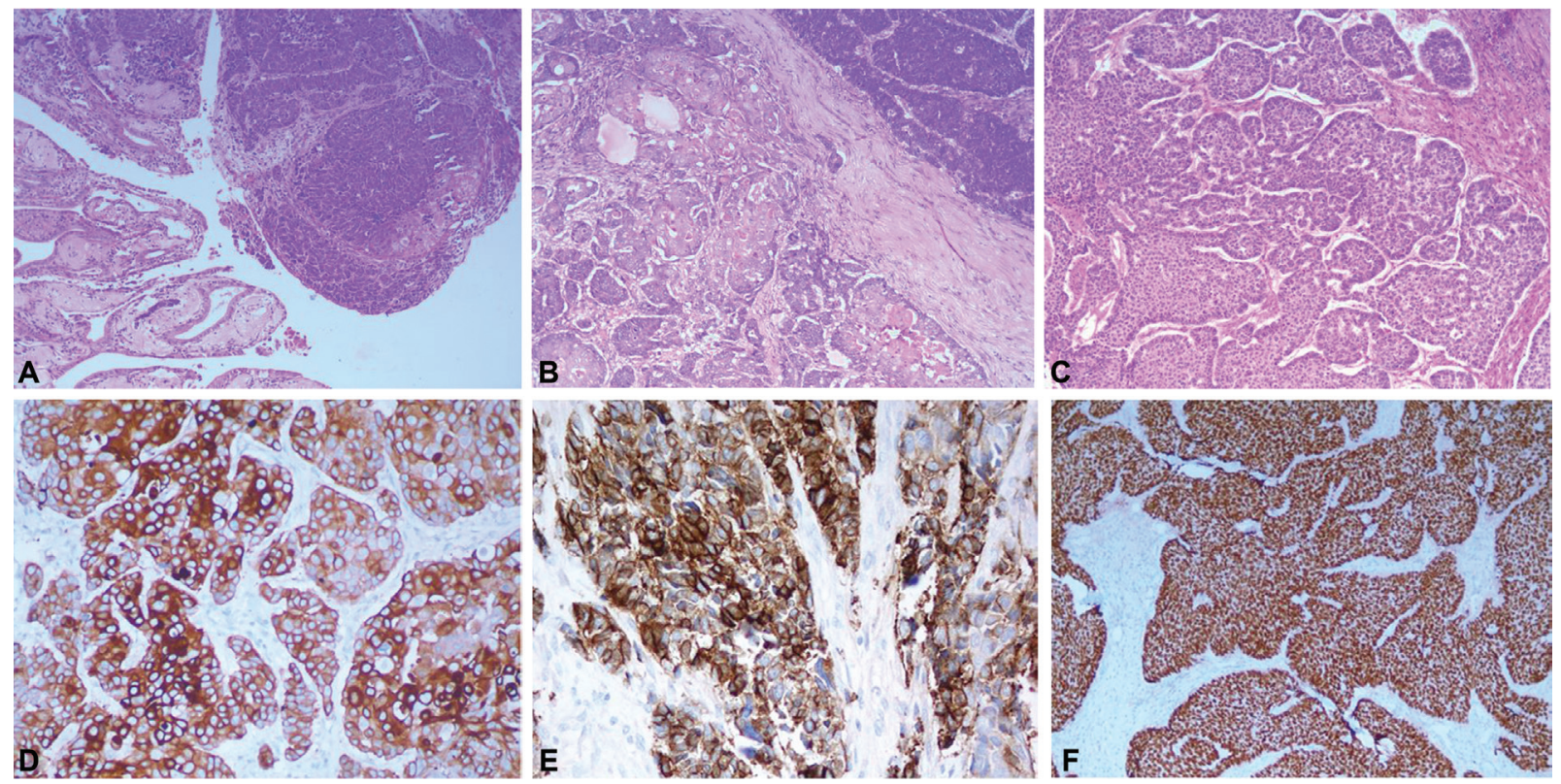

Fig. 5 (A-C) (hematoxylin and eosin, x100) and (D-F) (immunohistochemistry, x200). (A) Section from gall bladder shows a poorly differentiated tumor arising from its lining. (B) The tumor at places shows squamous differentiation with keratin pearl formation. (C) The infiltrating areas show tumor arrangement in nests, islands, and trabeculae with uniform round cells indicating neuroendocrine differentiation. (D) The squamous areas show diffuse and strong expression for high molecular weight cytokeratin. (E) The neuroendocrine differentiated areas show the expression of CD56. (F) The areas with neuroendocrine differentiation show diffuse nuclear expression for p63. 
showed neuroendocrine differentiation and was arranged in nests and chords. Brisk mitosis was seen ( $>20 / 10$ HPF). On immunohistochemistry ( $\mathbf{- F i g}$. 5D-F), the squamoid areas were found to be positive for $\mathrm{p} 63$ and $\mathrm{CK} 5 / 6$ and the neuroendocrine areas showed positivity for CD56 and synaptophysin. The tumor was negative for S-100, CK-7, and CK20.

\section{Discussion}

Adenocarcinomas (not otherwise specified) are the most common malignant GB neoplasms, accounting for $90 \%$ of all GB carcinomas. ${ }^{5}$ Papillary adenocarcinoma, intestinal adenocarcinoma, mucinous adenocarcinoma, signet-ring cell adenocarcinoma, and clear cell adenocarcinoma are all histological variants of adenocarcinoma. Squamous, small cell, adenosquamous, and undifferentiated carcinoma are the remaining epithelial cell types. ${ }^{6} \mathrm{NET}$, lymphomas, sarcomas, and metastases are examples of uncommon neoplasms. GB is an extremely rare site for neuroendocrine neoplasm, accounting for only 0.4 to $0.5 \%$ of all NETs. Only approximately $4 \%$ of GB neoplasms are NETs, with neuroendocrine carcinomas (NECs) accounting for an even smaller percentage. ${ }^{2,4}$ GBNETs, like GB carcinoma, are more common in women. ${ }^{7} \mathrm{NET}$ can occur alone or in combination with other types of GB cancers, such as adenocarcinoma or squamous cell carcinoma. ${ }^{8} \mathrm{~GB}$ is a rare location for neuroendocrine cells. The cause of GB NEC is debatable. ${ }^{8}$ The first is intestinal metaplasia of the GB mucosa, which can be caused by chronic inflammatory conditions and may contain neuroendocrine cells. Second, GBNETs can develop from endodermal stem cells or progenitor cells that have the ability to differentiate in multiple directions. Third, neometaplasia or trans-differentiation may result in adenocarcinoma differentiation that is abnormal, leading to NETs. Other than these, some authors believe that GB may be a site for heterotopic pancreatic tissue and NETs develop in this tissue. ${ }^{7}$

The presentation is usually with non-specific symptoms which might include upper abdominal pain, discomfort, weight loss, and jaundice.

According to previous research, the majority of them are nonfunctional, and the majority of lesions are discovered incidentally during the routine histological examination of post-cholecystectomy GB samples. ${ }^{4,9} \mathrm{Few}$ functional GBNETs may present with specific symptoms, with a very low reported incidence of carcinoid syndrome (1\%). According to the Surveillance, Epidemiology, and End Results program, a review of the pathological results of 41 GB-NETs (from 1973 to 2004) revealed that the majority of the tumors (89.7\%) were poorly differentiated or undifferentiated, only $2.4 \%$ were well-differentiated, and $7.3 \%$ were moderately differentiated. ${ }^{9}$ When compared with GB adenocarcinoma, NETs of GB usually have a poor prognosis. ${ }^{7}$

Preoperative diagnosis is infrequently made by radiological imaging because of low incidence and the similarity of the radiological findings to other GB tumors, specifically GB adenocarcinoma. ${ }^{2}$ Radiology case reports regarding GBNETs have described variable imaging features. However, in our case, the possibility of NET was considered preoperatively based on CT scan images, and on further NET work up, chromogranin was found to be elevated which was helpful for NET diagnosis. Chromogranin A is found to be elevated in $90 \%$ of gut NETs. The level of chromogranin correlates well with the tumor burden and recurrence and is an effective biology marker to suggest NET diagnosis. ${ }^{7}$

On ultrasound, it is indistinguishable from $\mathrm{Ca} G B$, and usually, a well-defined heteroechoic mass is seen in the GB fossa. ${ }^{4}$

There has been no reported difference in CT findings between adenocarcinoma and neuroendocrine carcinoma. ${ }^{8}$ NETs in other locations are typically hypervascular tumors. On contrast scans, primary NETs (gastrointestinal and lung NETs) and their metastases show hyperenhancement, and they are usually best seen in the arterial phase. ${ }^{10}$ Furthermore, primary hepatic NETs and metastatic NETs to the liver exhibit significant arterial enhancement, confirming their hypervascular nature, and may exhibit a washout pattern on venous/delayed phase images. ${ }^{11}$ This was observed in our case, which had a GB mass and a hepatic extension with arterial hypervascularity and relative washout. Lymph nodes (88\%), liver (88\%), lung (23\%), and peritoneum are the most commonly reported metastatic sites (19\%). ${ }^{7}$

Because NETs have overexpression of somatostatin receptors, somatostatin analog scintigraphy (SRS) is useful in the diagnosis of NETs. ${ }^{12}$ Positron emission tomography (PET)based SRS for NETs has high sensitivities, specificities, and accuracies. Recently, 68Ga positron-based PET, such as 68GaDOTANOC, 68Ga-DOTATOC, and 68Ga-DOTATATE, has become popular. Because the majority of NETs are metabolically inactive and do not take up 18-fluoro-deoxy-glucose (FDG), FDG PET plays a limited role. Only poorly differentiated NETs exhibit avid uptake of 18-FDG and FDG- PET, indicating their aggressive nature. ${ }^{10}$

\section{Management}

As with GB carcinoma, complete resection is the gold standard for GBNETs whenever possible. For pre-invasive and earlydetected cancers (T1), simple cholecystectomy is sufficient. Advanced lesions are typically treated with a combination of radical cholecystectomy and regional lymphadenectomy, as well as hepatic resection with adequate tumor-free margins. ${ }^{4}$

\section{Teaching Point}

An NET or NET differentiation should be considered if a GB tumor presents arterial enhancement with or without washout patterns on imaging. However, other neoplasms, such as advanced GB-adenocarcinomas, cholangiocarcinoma, hepatocellular carcinoma, or hepatic metastasis involving the GB, have a similar presentation. GB lymphoma is also associated with extensive lymph node enlargement.

\section{Conclusion}

If a GB tumor presents with or without hepatic extension and shows arterial enhancement with or without washout patterns at diagnosis, an NET or NET differentiation should be 
considered in differentials. The knowledge of NET of GB, either in pure form or along with other histological components, though a rare entity, is important.

\section{Ethical Approval}

Ethical clearance was taken from ethical committee. Informed consent was taken from the kin of the deceased.

\section{Conflict of Interest}

None declared.

\section{References}

1 Baxi AJ, Chintapalli K, Katkar A, Restrepo CS, Betancourt SL, Sunnapwar A. Multimodality imaging findings in carcinoid tumors: a head-to-toe spectrum. Radiographics 2017;37(02):516-536

2 Monier A, Saloum N, Szmigielski W, Alrashid A, Napaki SM. Neuroendocrine tumor of the gallbladder. Pol J Radiol 2015; 80:228-231

3 Mezi S, Petrozza V, Schillaci O, et al. Neuroendocrine tumors of the gallbladder: a case report and review of the literature. J Med Case Reports 2011;5:334

4 Eltawil KM, Gustafsson BI, Kidd M, Modlin IM. Neuroendocrine tumors of the gallbladder: an evaluation and reassessment of management strategy. J Clin Gastroenterol 2010;44(10): 687-695

5 Levy AD, Murakata LA, Rohrmann CA Jr. Gallbladder carcinoma: radiologic-pathologic correlation. [published correction appears in Radiographics 2001 May-Jun;21(3):766]Radiographics 2001; 21(02):295-314, 549-555

6 Yadav R, Jain D, Mathur SR, Sharma A, Iyer VK. Gallbladder carcinoma: an attempt of WHO histological classification on fine needle aspiration material. Cytojournal 2013;10:12

7 Yun SP, Shin N, Seo HI. Clinical outcomes of small cell neuroendocrine carcinoma and adenocarcinoma of the gallbladder. World J Gastroenterol 2015;21(01):269-275

8 Lee SW, Baek SY, Sung SH. Combined undifferentiated and neuroendocrine carcinomas of the gallbladder appearing as two separate lesions: a case report with radiological-pathological correlation. J Radiol Case Rep 2015;9(05):14-21

9 Hussain I, Sarvepalli D, Zafar H, Jehanzeb S, Ullah W. Neuroendocrine tumor: a rare, aggressive tumor of the gallbladder. Cureus 2019;11(09):e5571

10 Maxwell JE, Howe JR. Imaging in neuroendocrine tumors: an update for the clinician. Int J Endocr Oncol 2015;2(02):159-168

11 Wang LX, Liu K, Lin GW, Jiang T. Primary hepatic neuroendocrine tumors: comparing CT and MRI features with pathology. Cancer Imaging 2015;15(01):13

12 Tan $\mathrm{EH}$, Tan $\mathrm{CH}$. Imaging of gastroenteropancreatic neuroendocrine tumors. World J Clin Oncol 2011;2(01):28-43 